\title{
Forum CC
}

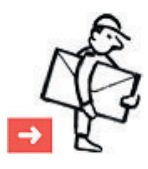

\section{Scepticisme croissant de la base à I'égard de la révision de la LAMal}

Les responsables de la FMH et des associations des médecins de premier recours accueillent favorablement la révision de la LAMal, qui prévoit entre autres l'introduction de réseaux de soins intégrés avec responsabilité budgétaire (RSIRB) et une quote-part différenciée. Les patients qui veulent conserver la liberté de choisir leur médecin se verront pénalisés par une quote-part deux fois plus élevée. Les cercles et les labels de qualité ne peuvent pas non plus vraiment conjurer le risque de rationnement consécutif à la médecine budgétaire et inhérent au nouveau système. Ils font tout au plus office de feuilles de vigne. Il n'existe aucune étude ni aucun modèle d'économie de la santé étayant l'hypothèse selon laquelle les RSIRB permettent d'économiser des coûts en garantissant un niveau de qualité au moins équivalent. Même des représentants de l'association des médecins de famille admettent qu'il ne sera pas possible, au cours de ces prochaines années, de prouver que l'organisation en réseau présente un meilleur rapport coût-efficacité que le libre choix du médecin. Il est à redouter qu'en cas d'absence de transparence, la médecine en réseau de soins déficitaire fera l'objet d'un subventionnement croisé par les bénéfices que les assurances auront accumulés avec le libre choix du médecin. De cette manière, les primes des patients avec libre choix du médecin seront artificiellement maintenues à un niveau élevé. Les malades chroniques sont considérés comme les principaux responsables des dépenses et la quote-part différenciée doit les pousser vers les RSIRB. Pour ces patients précisément, le risque de rationnement est considérable compte tenu de la pression budgétaire. Les investissements et les dépenses nécessaires au fonctionnement d'un RSIRB sont énormes et il est très légitime de se demander si leur compensation par les économies réalisées en contrepartie est possible sans rationnement. Nul ne l'ignore, les véritables facteurs de coûts ont des causes très différentes: le progrès médical et le vieillissement démographique. Contre eux, cette réforme est totalement impuissante. Notre élite a construit des circonstances qui, en réalité, n'existent pas. Et pourtant, nous serions en bonne position pour négocier compte tenu de la pénurie de médecins de premier recours qui s'annonce. Les collègues assidus sur le terrain commencent à se rendre compte que des nuages sombres se profilent à l'horizon. Le nombre croissant de lettres de lecteurs critiques visà-vis de la réforme témoigne de la défiance grandissante. Dans les plaidoyers des partisans de la réforme, des arguments tels que l'audace et le courage ne sont pas précisément de nature à favoriser la confiance. Les dépenses de santé continueront malgré tout à augmenter et les politiciens imputeront cette hausse à la défaillance du corps médical, en conséquence de quoi ils annuleront l'obligation de contracter ou introduiront une médecine étatique. Mais alors, pourquoi acceptons-nous cette mauvaise donne? La situation est symptomatique du conflit entre l'élite et la base. Pour sa stratégie, l'élite de la FMH n'a obtenu la bénédiction que de la Chambre médicale. Or, pour cette réforme fondamentale, une légitimation démocratique par la base aurait été nécessaire. Au lieu de se poser les bonnes questions, ce sont les membres hésitants qui sont traités de poules mouillées et d'étatistes. Les responsables des associations professionnelles se sont précipités pour faire des concessions aux politiciens sans consulter la base, ce qui restreint le champ d'introspection et l'horizon d'argumentation. Un débat démocratique au sein de la base n'est pas souhaité, car finalement, on ne veut pas perdre la face. Les relations au sein de la FMH sont mises à l'épreuve et le Comité central serait bien avisé de redevenir utile pour ses membres et de se montrer moins suffisant dans ses actes.

Dr René Haldemann, Richterswil

\section{Réponse}

Cher Collègue,

Merci de votre lettre à la rédaction, et de poser des questions importantes.

Il est nécessaire de préciser trois choses, je crois:

- Dans l'ensemble du projet de loi actuellement en discussion, il est question de coresponsabilité budgétaire, ce qui est toutà-fait différent d'une simple responsabilité budgétaire comme le serait un système de pure capitation. La co-responsabilité budgétaire signifie que le groupe de médecins engagé dans le réseau partage la responsabilité du budget avec les assureurs, dans une mesure à définir dans le contrat de réseau et qui est en général de l'ordre d'une dizaine de pourcents. Ce partage de responsabilité concerne évidemment aussi bien les économies réalisées que les dépassements budgétaires. Il est important de noter que ce ne sont jamais les médecins individuels qui assument cette responsabilité, mais bien le groupe.

Je ne vois là aucune raison d'imaginer que cela conduise à une sous-médication; outre l'éthique que l'on reconnaît au corps médical, le fait est que le budget est élaboré en commun, les médecins regroupés sont dans une position de force, l'expérience de chacun est prise en compte, et le vécu des réseaux existants est franchement positif. Concrètement, des réseaux de soins avec co-responsabilité budgétaire existent depuis 20 ans; actuellement, 50\% des généralistes suisses travaillent en réseau, dont 80\% avec co-responsabilité budgétaire il n'existe simplement aucun indice de rationnement dans ces réseaux (de même qu'il n'y a aucun indice de polypragmasie chez les confrères travaillant seuls!).

- Le projet de loi sur les soins intégrés contient une amélioration essentielle de la compensation des risques, selon la proposition faite en Commission par notre viceprésident Ignazio Cassis; selon cette disposition nouvelle, les maladies coûteuses, en particulier évidemment les maladies chroniques, feront l'objet d'une compensation qui fera définitivement disparaître la «chasse aux bons risques», respectivement le rejet des patients chroniques; ces patients pourront donc intégrer sans problème les réseaux - et ce sont assurément eux qui en profiteront le plus.

Permettez-moi d'ajouter encore ici que certes, la démographie et le développement technologique de la médecine continueront à faire augmenter les coûts, mais que justement, c'est une bonne raison pour tenter de les modérer un peu; même si le système des soins intégrés vise à la qualité la meilleure avant de viser des économies, on sait que le «Managed care» coûte en fin de compte moins cher ... et oui, les études nécessaires sont disponibles! Je citerai par exemple K. Beck: Effizienzsteigerung dank Managed Care. Datamaster. 2009;5:15-21; ou du même auteur et U. Käser: Nachhaltigkeit von Kosteneinsparungen in Managed-Care-Modellen. Care Management. 2008;1: Nr. 5.

- La FMH dispose d'une structure réellement démocratique, où chaque membre peut 
élire ses représentants à l'échelon «supérieur»; ces structures (Chambre médicale, Assemblée des délégués, Comité central) sont là pour prendre, chacune selon ses compétences, les décisions nécessaires au bon fonctionnement de l'organisation et à une bonne représentation du corps médical et de ses intérêts.

Je ne pense pas qu'il soit juste, dans le fonctionnement démocratique d'une société, de mettre en cause la représentativité des organes élus chaque fois que leurs décisions indisposent. La démocratie demande l'adhésion active de chacun-e, autrement dit le respect des institutions et des déci-

sions prises, sous peine de perdre cohérence, cohésion et crédibilité; et dans le long terme, au-delà des divergences et des déceptions ponctuelles, les intérêts de tous se retrouvent assurément pris en compte.

Dr Jacques de Haller, Président de la FMH

\section{Courrier au BMS}

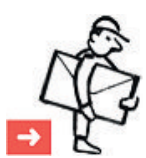

Effizienzsteigerung dank Managed Care? Die Frage stammt von PD Dr. Konstantin Beck, Leiter des CSS Instituts für empirische Gesundheitsökonomie, teilweise finanziert durch Mittel der CSS Kranken-Versicherung AG. Ich zitiere zwei Schlussfolgerungen aus dieser Arbeit:

1. Auf den ersten Blick, das muss man sich ehrlicherweise eingestehen, sind Spareffekte unter 10\% angesichts einer 19-jährigen Erfahrung in diesen Modellen ernüchternd wenig.

2. Mittelfristig werden die Verträge mit mässig performenden MC-Anbietern gekündigt werden und die Kunden in die besseren $\mathrm{HMO}$ am Ort gesteuert.

Meine Folgerungen:

1. Der Spareffekt der MC-Modelle ist bei weitem nicht so gross, wie immer behauptet wird.

2. Die Versicherer werden das Sagen haben und mit ihrem rein ökonomisch-materialistischen Denken knallhart Patienten und Ärzte dorthin dirigieren, wo es ihnen passt. Es ist klar, dass das Arzt-PatientenVerhältnis leiden und sich verschlechtern wird, weil ökonomische Faktoren (Performance) unseren ärztlichen Alltag bestimmen werden. Dies alles mit dem Wissen dass weder die Gesundheitskosten wesentlich gebremst, noch die Qualität der ärztlichen Arbeit, noch rationelles Arbeiten verbessert werden, wie Santésuisse dies behauptet.
Es ist zu hoffen, dass unsere Politiker diesen Artikel* lesen und die entsprechenden Schlüsse daraus ziehen.

Dr. med. Martin Sutter, Worb

* Im Internet nachzulesen unter: www.css-institut.ch/gzd2_beck_d509.pdf

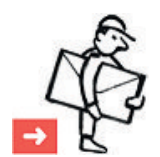

Die Musik spielt in Bern, man spielt mit oder hört zu

Verbandsarbeit heisst politische Arbeit. Politische Arbeit ist dann erfolgreich, wenn sie Mehrheiten bzw. Entscheide zustande bringt. Das gelingt bei maximaler Vernetzung, optimalem Informationsstand und gewiefter Taktik. Von dieser Prämisse ausgehend, ist die Intention des Präsidenten der FMH, Jacques de Haller, sich im 2011 für einen Sitz im Parlament $\mathrm{zu}$ bewerben, nur $\mathrm{zu}$ begrüssen (vorausgesetzt, alles sei wohldurchdacht). Wer das verneint, verkennt die Entscheidstrukturen oder reagiert im Kurzschluss ab der Intention de Haller's, seine Ambitionen bei den Linken zu lancieren.

Als Liberaler gibt auch mir die Tendenz zu denken, dass unser Berufsstand, per se ein freier, politisch immer mehr nach links rutscht hin zu Positionen, welche gesellschaftliche und wirtschaftliche Probleme lösen wollen, indem sie auf staatliche Regelungen setzen (vulgo: etatistisch). Aber auch hier gilt es vor den eigenen politischen Präferenzen die Fakten zu beurteilen. Und diese zeigen, dass die SP eine gewichtige Playerin

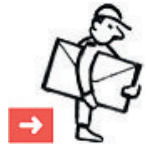

\section{Korrigendum}

Die in der SÄZ Nr. 34 angegebene Internetadresse für das Diskussionsforum des Vereins für freie Arztwahl [1] muss korrigiert werden. Die richtige Adresse lautet www.vfa.forencity.ch. Auf die Homepage des Vereins gelangt man via www.verein-freie-arztwahl.ch

1 Bracher D, Colombo S. Aufruf, dem Verein für freie Arztwahl beizutreten. Schweiz Ärztezeitung. 2010;91(34):1304. ist im Gesundheitswesen. Zum Glück nicht alleine, auch die FDP hat gewichtige Akteure bei verschiedenen Leistungserbringern, bspw. Charles Favre für die Spitäler, Claude Ruey für die Kassen und last but not least Ignazio Cassis als Vizepräsident der Ärzteschaft.

Was könnte also besseres geschehen, um die Politik der Ärzteschaft aktiv mitzugestalten, als den Präsidenten und den Vize-Präsidenten dort zu haben, wo die Musik spielt, im nationalen Parlament, ob einem die Musik nun gefällt oder nicht?

Dr. med. Walter Bär, Schönbühl-Urtenen

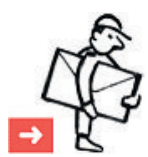

\section{Hausärztliche Differentialdiagnose ...}

\section{Diagnose: Lingua bifida}

(gespaltene Zunge)

Beurteilung: Anamnese und Befunde ergeben den dringenden Verdacht, dass Politik, santésuisse und FMH (u.v.m.) den Weiterbestand des Hausarztes gar nicht mehr wollen, obwohl vor Bildschirmen, Mikrofonen und Artikelschreibern das Gegenteil gepriesen wird! Differentialdiagnostisch käme allenfalls auch eine Schizophrenia simplex in Frage; das offensichtlich bestrebende und zielgerichtete Verhalten der Erwähnten lässt leider die erste, schlimmere Diagnose vermuten.

Procedere: Die Therapiemöglichkeiten sind leider beschränkt (Wahlen, Referenden, Streiks, ...), und in Folge zunehmender Resistenzentwicklung und gefährlicher Nebenwirkungen nicht ohne Risiko! Ein Behandlungs- 